\title{
Metodología para la formulación de proyectos de recuperación química mediante analogías
}

\author{
Piter Andrés Vega Vanegas ${ }^{1 *}$; Teddy Yulian Zuñiga Ruiz¹; Freddy Humberto Escobar Macualo; \\ Camilo Andres Guerrero Martin² \\ ${ }^{1}$ Escuela de Ingeniería de Petróleos. Universidad Surcolombiana (USCO). Avenida Pastrana Cra 1. Huila, Colombia. \\ ${ }^{2}$ Instituto de Macromoléculas. Universidad Federal de Rio de Janeiro (UFRJ). Calle. Ciudad Universitaria. \\ Rio de Janeiro, Brasil.
}

Email: piandru96@hotmail.com

\begin{abstract}
Resumen
En las últimas décadas la recuperación terciaria, especialmente en la que intervienen productos químicos, ha sido materia de investigación por la academia y la industria, su capacidad para reducir la saturación de petróleo residual $\left(\mathrm{S}_{\mathrm{or}}\right)$ e incrementar el factor de recobro de un yacimiento, le hacen ser un método altamente atractivo y objeto de inversión. La identificación de campos análogos es un paso importante en la planificación de un proyecto CEOR por sus siglas en inglés (Chemical Enhanced Oil Recovery), especialmente cuando existe a nivel mundial poca información de campos costa afuera que hayan implementado este tipo de métodos. Este artículo propone y evalúa una metodología con la finalidad de establecer si un campo costa afuera ubicado en la cuenca de Santos, Brasil; es un buen candidato o no para ser sometido a inyección de polímeros. La metodología propuesta se define de cuatro pasos: procesamiento de datos recopilados de la búsqueda de información a nivel mundial, selección de campos análogos con base en el screening propuesto por Paris de Ferrer (2001), uso de un modelo estadístico y clasificación de similitud. En primera instancia se realiza un análisis y procesamiento de la base de datos disponible de los campos costa afuera que han implementado la técnica, seguidamente se identifican las principales propiedades de roca y fluido que describen cada campo. Posteriormente, las propiedades del campo objetivo y los encontrados en la base de datos son comparados por medio de un modelo estadístico y finalmente se procede a la clasificación, a través de una ponderación según su analogía respecto al campo objeto de estudio.
\end{abstract}

Palabras clave: Analogía, recuperación química mejorada de petróleo, base de datos, costa afuera.

\section{Methodology for a formulation of chemical recovery projects through analogies}

\begin{abstract}
In recent decades, tertiary recovery, especially involving chemical products, has been area of research by the academy and industry. Its ability to reduce the saturation of residual oil (Sor) and increase the recovery factor of a reservoir, make it a highly attractive and worth investing method and. The identification of analogous fields is an important step for planning a CEOR project by its acronym in English (Chemical Enhanced Oil Recovery), especially when there is few information on offshore fields worldwide that have implemented this type of method. This article proposes and evaluates a methodology with the purpose of establishing if an offshore field located in the Santos basin Brazil is a good candidate or not to be subjected to polymer injection. The proposed methodology is defined by four steps: processing of data collected from the search for information worldwide, selection of analogous fields based on the screening proposed by Paris de Ferrer (2001), use of a statistical model and classification of similarity. In the first instance, an analysis and processing of the available database of the offshore fields that have implemented the technique is carried out, then the main properties of rock and fluid that describe each field are identified. Subsequently, the properties of the objective field and those found in the database are compared by means of a statistical model and finally the classification is carried out, using a weighting according to its analogy with respect to the field under study.
\end{abstract}

Keywords: Analogy, chemical enhanced oil recovery, data base, off-shore.

Cita: Vega Vanegas, P. A., Zuñiga Ruiz, T. Y., Escobar Macualo, F. H. \& Guerrero Martin, C. A. (2019). Metodología para la formulación de proyectos de recuperación química mediante analogías. Revista Fuentes: El reventón energético, 17(1), 29-35. 


\section{Introducción}

Una tecnología EOR eficiente que busca controlar el problema de fingering generado en la inyección de agua es la inyección de polímeros, que tiene como objetivo mejorar la relación de movilidad entre la fase desplazante y la fase desplazada, aumentando así la eficiencia de barrido para lograr un mayor porcentaje de petróleo recuperado (Pinto et al, 2018). Las relaciones de movilidades adversas entre el agua y el aceite y la alta heterogeneidad de algunas formaciones geológicas hacen necesario el uso de los polímeros como agentes de control de movilidad (Aya et al, 2018). Durante los últimos años, varias actividades de investigación han identificado varios tipos de polímeros tanto naturales como sintéticos para su uso en recobro mejorado. Los desarrollos recientes que logran acomodarse a la gran variedad de condiciones que se encuentran en los campos petrolíferos alrededor del mundo, incluyen poliacrilamidas y biopolímeros (Sun et al, 2018).

La identificación de campos análogos es importante en la planificación para implementar CEOR, especialmente cuando existen a nivel mundial pocas aplicaciones y experiencia en campos costa afuera (Díaz et al, 2017). Esta investigación permite por medio de analogías y un modelo estadístico conocer la viabilidad de la inyección de polímeros como método de recobro mejorado en la cuenca de Santos de Brasil, teniendo en cuenta los resultados de dicho método aplicado a otros campos petroleros con base en información extraída de fuentes mundiales que describe propiedades de la roca y del fluido de los campos. La clave está en caracterizar cada campo mediante un conjunto de propiedades que se puedan usar para compararse con las propiedades de un campo objetivo, mediante el uso de la teoría de la probabilidad y la información (Toro et al, 2018).

El estudio basado en casos de la literatura consiste en la búsqueda de campos que sean semejantes, es decir, potencialmente buenos análogos, pero que necesariamente no coincidan en su totalidad con algún parámetro en particular. Aquí se presenta un resumen de los campos análogos; en total se revisaron 12 campos de los cuales se eligieron siete que disponían de toda la información requerida para llevar a cabo la implementación de la metodología propuesta.

\section{Fundamentación conceptual inyección de polímeros}

Es un proceso de recobro mejorado que se desarrolló para mejorar la inyección de agua convencional. Esta técnica consiste en aumentar la viscosidad del agua y de esta manera reducir la permeabilidad relativa de la roca al agua, es decir, disminuye la razón de movilidad del agua hasta cerca de la unidad o incluso menos, con el fin de lograr un barrido más uniforme del yacimiento y a su vez lograr un mayor desplazamiento de petróleo. (Molano, Muñoz y Díaz, 2014) (Jimenez A.M., 2009)

\section{Clasificación de los polímeros}

Actualmente estos productos químicos se clasifican como naturales, semi-sintéticos y sintéticos; los naturales o biopolímeros tienen origen en la naturaleza y los seres vivos, mientras que los sintéticos se crean a partir de procesos industriales con base en elementos propios de la naturaleza (Araujo et al, 2018). Los polímeros semi-sintéticos por otra parte, son producto del manejo químico de los biopolímeros.

\section{Analogía}

Permite comparar dos o más cosas y de esta manera establecer relaciones entre objetos, conceptos o experiencias. Con las analogías se busca encontrar semejanzas entre los datos de proyectos efectuados a nivel mundial y los datos de un campo prospecto, con el fin de estudiar e implementar la inyección de polímeros en un campo si los resultados fueron óptimos.

Factores que afectan el flujo del polímero a través del medio poroso: Para el diseño del bache de inyección es muy importante considerar factores como la salinidad, viscosidad, temperatura, adsorción, presencia de acuífero y capa de gas, además de la etapa de inyección de agua puesto que cambios en alguno de estos factores se traduce en la alteración de la solución polimérica en el yacimiento (Hernández et al, 2018; López et al, 2017).

\section{Metodología}

El diagrama de flujo que se encuentra en la Figura 1 describe la secuencia básica de eventos que se generan para una formulación de proyectos de recuperación química mediante analogías. Primero se selecciona un campo prospecto teniendo en cuenta los parámetros según Paris de Ferrer, luego se buscan los campos costa afuera que han implementado la inyección de polímeros a nivel mundial, seguidamente se encuentran las propiedades que los describen para así almacenar la información en una base de datos, posteriormente se calculan los factores de similitud Fi, Ii y Zi para encontrar el grado de importancia de cada parámetro analógico de cada campo y finalmente se halla el puntaje de todos los campos de la base de dato. 


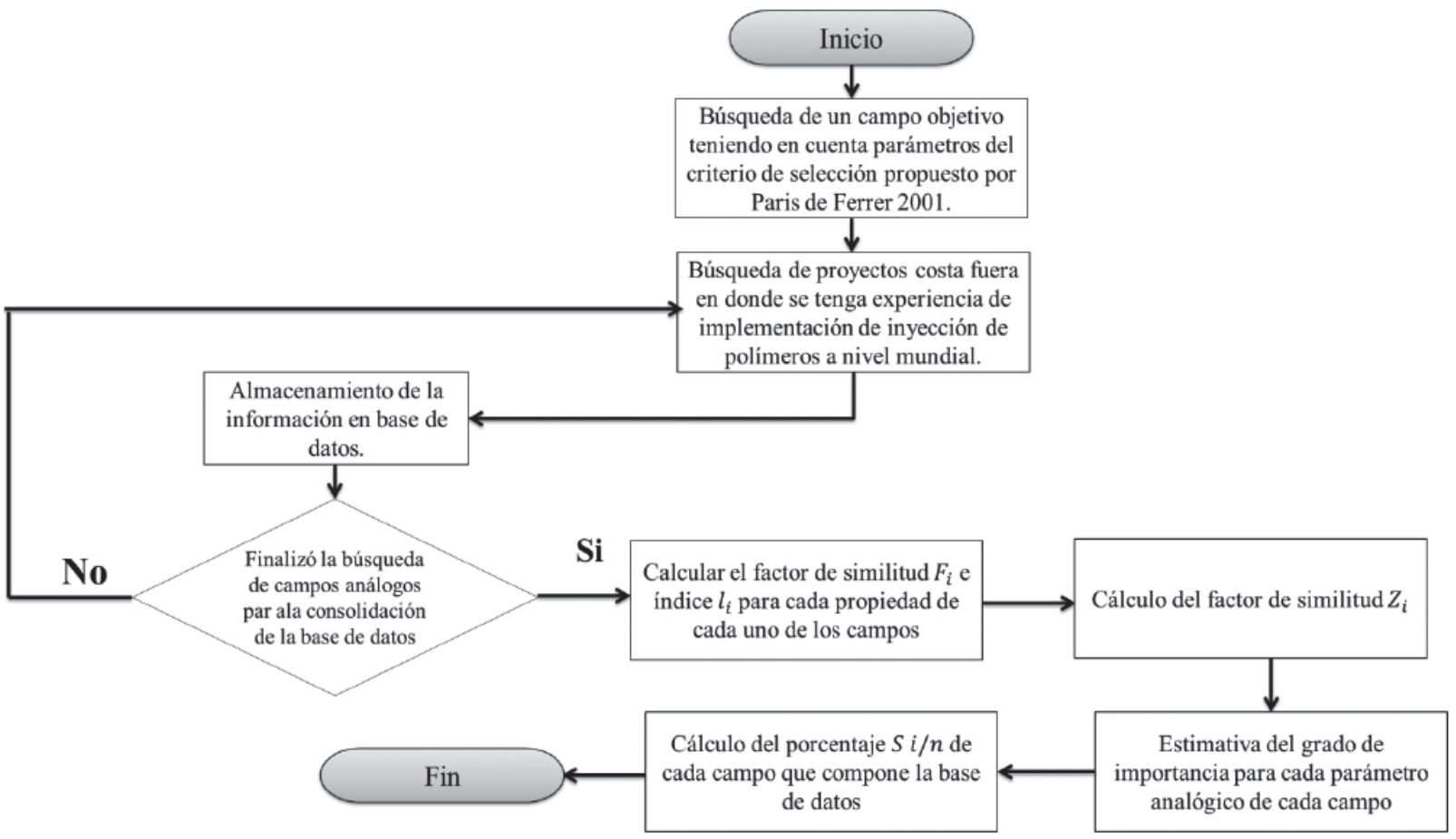

Figura 1. Flujograma de la metodologia

La metodología propuesta a continuación se basa en herramientas básicas de estadística tomando como referencia el proyecto de grado "Análisis e Interpretación de Yacimientos Sometidos a Inyección de Químicos (Surfactantes, Polímeros y Miscelares) Mediante Analogías", por Jiménez (2009), donde se desarrolló un modelo estadístico incluyendo un factor de similitud (Fi), el proyecto de grado "Evaluación de Yacimientos Prospectos para la Implementación de Procesos de Combustión In-situ Mediante Analogías”, por Palacio (2010), donde se establece un peso relativo o grado de importancia $\left(\mathrm{W}^{*} \mathrm{nj}\right)$ de acuerdo con la dispersión de datos, a cada parámetro clave para el desarrollo de esta analogía y el proyecto de grado "Diseño del Completamiento y del Sistema de Levantamiento Artificial en un Pozo con Recobro por Combustión In-situ Mediante Analogías", por Alarcón y Navarro (2014), donde se desarrolla un índice de similitud Ij i/n.

\section{Modificación al modelo}

El modelo estadístico que se desarrolló en los trabajos anteriores permite llevar a cabo exitosamente analogías entre los campos de una base de datos y el campo de estudio, no obstante, cuando se tienen rangos de valores en la base de datos, el factor de similitud Fi tiene en cuenta el valor más cercano al dato del campo de estudio, discriminando así la media de los datos y causando mayor dispersión en el resultado.
Por lo anterior se sugiere una modificación al modelo implementando un nuevo factor de similitud que relacione el factor $\mathrm{Fj} \mathrm{i} / \mathrm{n}$ y el índice $\mathrm{Ij} \mathrm{i} / \mathrm{n}$ con el objetivo de tomar en cuenta tanto los valores de los rangos más cercanos al campo de estudio como la media del rango y de esta manera obtener un resultado más certero.

A continuación, se muestra cómo se realizó la metodología propuesta:

1. Fue seleccionado un campo objetivo costa afuera, denominado campo X de la cuenca de Santos teniendo en cuenta los parámetros en el Screening propuesto por Paris de Ferrer que se encuentran en la tabla 1. En la tabla 2 se encuentran las principales características de este campo.

Tabla 1. Parámetros de Screening.

\begin{tabular}{|c|c|}
\hline \multirow{2}{*}{ Parámetros del yacimiento } & Autor \\
\cline { 2 - 2 } & Magdalena (2001) \\
\hline Gravedad API & $>25$ \\
\hline Viscosidad del aceite (cP) & $<150$ \\
\hline Espesor (m) & NC \\
\hline Permeabilidad promedio (mD) & $>20$ \\
\hline Temperatura (K) & $<354$ \\
\hline
\end{tabular}


Tabla 2. Datos del campo objetivo.

\begin{tabular}{|c|c|}
\hline \multicolumn{2}{|c|}{ Campo X } \\
\hline Parámetros de los Fluidos del Yacimiento \\
\hline Gravedad API & 28 \\
\hline Viscosidad (cP) & 1.14 \\
\hline Parámetros del Medio Poroso \\
\hline Porosidad (Fracción) & 0.2 \\
\hline Permeabilidad (mD) & 1000 \\
\hline Temperatura (K) & 353 \\
\hline Profundidad (m) & 5000 \\
\hline Espesor (m) & 30 \\
\hline
\end{tabular}

2. Fue elaborada una base de datos y se seleccionaron los campos costa afuera que utilizaron la inyección de polímeros como método de recobro mejorado a nivel mundial, esta base de datos almacenó parámetros que caracterizaban los campos como: porosidad (fracción), permeabilidad $(\mathrm{mD})$, temperatura de yacimiento $(\mathrm{K})$, gravedad API, viscosidad del crudo (cP), profundidad del yacimiento $(\mathrm{m})$ y espesor neto $(\mathrm{m})$. Algunos autores tomados como referencia para obtener los valores de los parámetros de los campos fueron: Dovan (1990), Reid (1996), Osterloh (1998), Xiang (2011), Selle (2013), Jones (2015), Morel (2015) y Rimoldi (2017). (Ver ecuación 1) La tabla 3 en el anexo 1 permite apreciar los parámetros que caracterizan los campos análogos seleccionados de la base de datos.

$$
W_{n j}^{*}=\frac{W_{j}^{*}}{\sum W_{j}^{*}}
$$

3. Fue calculado el grado de importancia para cada propiedad de cada campo de la base de datos por medio de los criterios estadísticos como: media, desviación estándar, coeficiente de variación de Pearson y la respectiva formula del peso. El grado de importancia se calculó con la ecuación 1, donde $\mathrm{Wj}$ hace referencia al peso global del parámetro j, que se utiliza para la realización de la analogía. Se realiza esta normalización para obtener una distribución mejor de cada peso global.

4. Fue calculado el factor de similitud Fi propuesto por Jiménez (2009), por medio de la tabla 4, (anexo 1), esta tabla mostró las diferentes ecuaciones de Fi dependiendo de la condición. Donde pi es el valor de la propiedad que se va a comparar y que pertenece a cada campo de la base de datos, pie es el valor de la propiedad a comparar y que pertenece al campo objetivo y max (|pi - pie|) es la máxima diferencia que se encuentra comparando cada uno de los campos de la base de datos con el campo de estudio respecto a una misma propiedad.

5. Fue calculado el índice Ii (Alarcon y Navarro, 2014) con la ecuación 2, Donde $J_{n}$ es el parámetro del campo de estudio y Ji es el parámetro del campo de la base de datos. El valor del índice $I_{j i / n}$ entre más cerca esté de 1 , indicara que es más semejante y entre más se acerque a cero, indicara que hay un grado de diferencia mayor.

$$
I_{j i / n}=1-\left|\frac{J_{n}+J_{i}}{J_{n}-J_{i}}\right|
$$

6. Se halló el nuevo factor de similitud Zi combinando el factor Fi y el índice Ii por medio de una media aritmética como se muestra en la ecuación 3.

$$
Z_{j i / n}=\frac{F_{j i / n}+I_{j i / n}}{2}
$$

7. Finalmente se obtuvo el puntaje (valor porcentual) de cada campo de la base de datos respecto al campo objetivo con la ecuación 4.

$$
S_{i / n}=\sum_{j=1}^{j=m}\left(W_{j} * Z_{j i / n}\right)
$$

\section{Resultados}

Se aplicó con éxito el método propuesto, obteniendo 7 campos análogos. En la figura 2 se observan los campos con los puntajes de similitud respecto al campo objetivo, los tres campos más análogos fueron el campo Heidrun, el campo Belayim y el campo Dalia. El campo Heidrun resultó ser el campo más análogo con un puntaje general de $80.20 \%$, debido a su semejanza en parámetros como gravedad API, viscosidad y espesor con relación al campo $\mathrm{X}$; aunque sus parámetros porosidad y permeabilidad varían de forma moderada respecto a estos mismos parámetros del campo X. Por otra parte se tiene el campo Belayim con un puntaje general de $72.57 \%$ en donde algunos de sus parámetros se asemejan a los del campo X, aunque difiere bastante en parámetros como gravedad API, viscosidad y espesor. Finalmente, está el campo Dalia con un puntaje general de $68.90 \%$ con parámetros similares respecto a los del campo X como permeabilidad y porosidad. (Han, M. et al 2006)

Es importante destacar que únicamente se tienen en cuenta las operaciones llevadas a cabo en campos costa fuera, dada la complejidad del procedimiento, además de contar con el recurso de agua salada para la formulación del bache de inyección. 


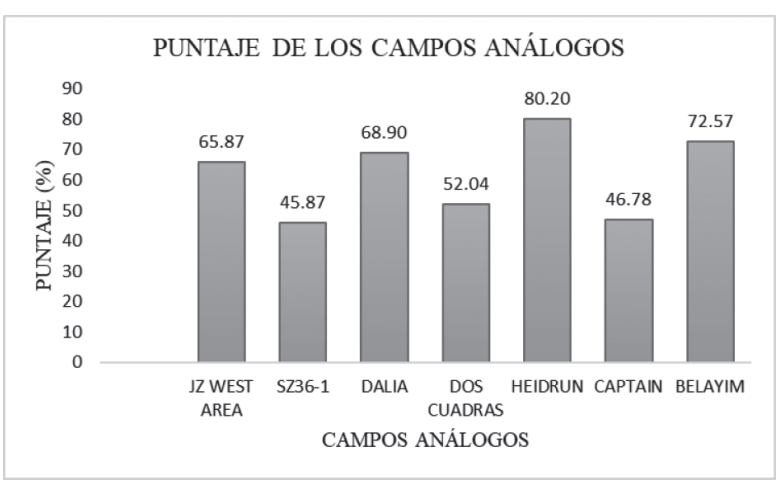

Figura 2. Puntaje de los campos análogos.

\section{Conclusiones}

Se realiza una propuesta para la inyección de polímeros como método recuperación mejorada aplicada en la cuenca de Santos, Brasil con base en los análisis técnicos previos y se identifican los principales trabajos de inyección polímeros aplicados a campos costa afuera a nivel mundial.

Fue desarrollada una base de datos con información encontrada históricamente de todos los campos costa afuera que utilizaron la inyección de polímeros como método de recobro mejorado a nivel mundial, entre los datos seleccionados se incluyen propiedades que caracterizan cada campo, como porosidad, permeabilidad, temperatura del yacimiento, gravedad API, viscosidad del aceite, profundidad del yacimiento y espesor neto, esta base de datos permite el análisis de la viabilidad de la inyección de polímeros en campos costa afuera semejantes, comparando un campo de estudio con características de otros campos. En consonancia con lo anterior, se concluye que la organización de datos es un paso primordial para la generación de analogías y construcción de conocimiento.

La dificultad que se presentó durante la realización de este artículo, radicó en encontrar los valores de los parámetros para cada campo de la base de datos.

Considerando que uno de los factores críticos en el estudio de las analogías por medio de este modelo estadístico es el número de parámetros analógicos utilizados, se recomienda el uso de la mayor cantidad de ellos como sea posible. Aunque la información disponible en la base de datos no permitió usar parámetros como el factor de recobro, se recomienda en futuros estudios de procesos EOR, involucrar además de este, todos aquellos parámetros que afectan directamente la recuperación mejorada.
El estudio de los campos análogos con base en conceptos estadísticos es una herramienta de gran utilidad para realizar la caracterización preliminar y obtener conocimientos sobre el desarrollo de campos nuevos y complejos donde la información es limitada. No solo se utiliza esta metodología para procesos de recobro químico en campos costa afuera, sino que también puede ser aplicada en cualquier campo en tierra.

Mediante el uso del modelo estadístico y el desarrollo de analogías se identificaron los tres campos más análogos al campo de estudio; el campo Heidrun seguido del campo Belayim y finalmente el campo Dalia, proyectos en los cuales el polímero seleccionado fue una poliacrilamida capaz de resistir las condiciones del yacimiento.

Se debe precisar que la consistencia de la información usada para el desarrollo de cualquier análisis técnico debe ser alta, es decir, que tanto las variables cualitativas como las variables cuantitativas deben ser extraídas de fuentes confiables, que permitan ratificar la certeza de los resultados y en el caso de la inyección de polímeros como método EOR, que permitan inferir la viabilidad de su aplicación en un campo de estudio, a partir de unos campos análogos. Es por lo anterior que se recomienda el uso de plataformas especializadas que respondan a las necesidades que demandan el desarrollo de análisis técnicos para procesos EOR.

Por medio de la estadística se elabora una evaluación de los datos existentes en la base de datos de campos costa afuera que han implementado la inyección de polímeros a lo largo de la historia. De esta forma, se calcula un puntaje que funciona como un índice de similitud para cada campo de la base de datos respecto al campo objetivo.

\section{Bibliografía}

1. Alarcón, J. E., y Navarro, S. A. (2014). Diseño del Completamiento y del Sistema de Levantamiento Artificial en un Pozo Con Recobro por Combustión In-Situ Mediante Analogias. Bucaramanga, Colombia: Universidad Industrial de Santander.

2. Araujo, Y. C., \& Araujo, M. (2018). Polymers for application in high temperature and high salinity reservoirs-critical review of properties and aspects to consider for laboratory screening. Revista Fuentes, 16(2).

3. Aya, C. L. D., Guardia, V. M. D., Toro, G. A. M., García, R. H. C., \& Pérez, H. I. Q. (2018). Metodología para la priorización de tecnologías 
emergentes de recobro mejorado químico. Revista Fuentes, 16(2).

4. Díaz, R. J., Navarro, S. F. M., \& Tavera, C. P. S. (2007). Modelo estadístico para la realización de analogías orientadas a procesos de recobro mejorado. Revista Fuentes, 5(1).

5. Dovan, H. T., Hutchins, R. D., y Terzian, G. A. (1990). Dos Cuadras Offshore Polymer Flood. Society of Petroleum Engineers. SPE-20060-MS.

6. Han, M., Xiang, W., Zhang, J., Jiang, W., y Sun, F. (2006). Application of EOR Technology by Means of Polymer Flooding in Bohai Oilfields. Society of Petroleum Engineers. SPE-104432-MS.

7. Hernandez, F. A. T., Niño, J. C. L., \& Moreno, R. L. (2018). Effects of salts and temperature on rheological and viscoelastic behavior of low molecular weight HPAM solutions. Revista Fuentes, 16(1), 19-35.

8. Jiménez, A. M. (2009). Análisis e Interpretación de Yacimientos Sometidos a Inyección de Químicos (Surfactantes, Polímeros y Miscelares) Mediante Analogías. Bucaramanga, Colombia: Universidad Industrial de Santander.

9. Jones, C., Ross, M., Getliff, J., Fuller, M., Hiscox, I., y Mandracchia, F. (2015). Captain Field Injector Performance, Historical Perspective and Recent Improvements. Society of Petroleum Engineers. SPE-174183-MS.

10. Lazzarotti, M., Rimoldi, A., Clementi, A., Mawad, M., y Abd Elrahman, M. (2017). Belayim Land - Polymer Injection Pilot Project. Offshore Mediterranean Conference. OMC-2017-722.

11. López, D. A., León, J. M., Cabrera, F., \& Manrique, E. (2017). Evidences of CDG formation and possible interpretations of core flood studies. Fuentes: El reventón energético, 15(2), 31-47.

12. Molano, A. M. J., Navarro, S. F. M., \& Díaz, R. J. (2014). Metodología para el diseño de baches en un proceso de inyección de polímeros para recobro mejorado, considerando fenómenos de interacción roca/fluidos. Fuentes: El reventón energético, 12(2), 6.

13. Morel, D. C., Zaugg, E., Jouenne, S., Danquigny, J. A., y Cordelier, P. R. (2015). Dalia/Camelia
Polymer Injection in Deep Offshore Field Angola Learnings and In Situ Polymer Sampling Results. Society of Petroleum Engineers. SPE- 174699-MS.

14. Osterloh, W. T. (1998). Polymer Transport and Theological Properties for Polymer Flooding in the North Sea Captain Field. Society of Petroleum Engineers. SPE-39694-MS.

15. Palacio, C. A. (2010). Evaluación de Yacimientos Prospectos para la Implementación de Procesos De Combustión In Situ Mediante Analogías. Bucaramanga, Colombia: Universidad Industrial de Santander.

16. Paris de Ferrer, M. (2001). Inyección de agua y gas en yacimientos petrolíferos. Maracaibo, Venezuela: Ediciones Astro Data SA.

17. Pinto, M. S., Herrera, D. M., \& Angarita, J. C. G. (2018). Production optimization for a conceptual model through combined use of polymer flooding and intelligent well technology under uncertainties. Revista Fuentes, 16(1), 37-45.

18. Reid, B. E., Høyland, L. A., Olsen, S. R., y Petterson, O. (1996). The Heidrun Field Challenges in Reservoir Development and Production. Offshore Technology Conference. OTC-8085-MS.

19. Selle, O. M., Fischer, H., Standnes, D. C., Auflem, I. H., Lambertsen, A. M., Svela, P. y Melien. (2013). Offshore Polymer/LPS Injectivity Test with Focus on Operational Feasibility and Near Wellbore Response in a Heidrun Injector. Society of Petroleum Engineers. SPE- 166343 -MS.

20. Sun, G., Crouse, B., Freed, D. M., Xu, R., Bautista, J., Zhang, R., ... \& Dressler, M. (2018). Polymer flooding-Does Microscopic Displacement Efficiency Matter?. Revista Fuentes, 16(2).

21. Toro, G. M., Herrera, J. J., Orrego, J. A., Rojas, F. A., Rueda, M. F., \& Manrique, E. J. (2018). Effect of ionic composition in water: oil interactions in adjusted

22. Xiang, W., y Zhou, W. (2011). Field Experience of Produced Polymer Control in Offshore Oilfield. Society of Petroleum Engineers. SPE144277-MS.

Recepción: 11 de junio de 2018

Aceptación: 06 de junio de 2019 


\section{Anexo 1}

Tabla 3. Lista de campos análogos.

\begin{tabular}{|c|c|c|c|c|c|c|c|c|}
\hline \multirow{2}{*}{ Región } & Campo & $\begin{array}{c}\text { Porosidad } \\
\text { (Fracción) }\end{array}$ & $\begin{array}{c}\text { Permeabilidad } \\
(\mathbf{m D})\end{array}$ & $\begin{array}{c}\text { Temperatura } \\
\text { B(K) }\end{array}$ & $\begin{array}{c}\text { Gravedad } \\
\text { API }\end{array}$ & $\begin{array}{c}\text { Viscosidad } \\
\text { Crudo (cP) }\end{array}$ & $\begin{array}{c}\text { Profundidad } \\
\text { (Mts) }\end{array}$ & $\begin{array}{c}\text { Espesor } \\
\text { (Mts) }\end{array}$ \\
\hline & JZ West & $0.22-0.36$ & $10-5000$ & 330.15 & $17-22$ & 17.1 & $2300-2500$ & 23.3 \\
\cline { 2 - 9 } & SZ36-1 & $0.28-0.35$ & 2.63 & 338.15 & $11-19$ & 70 & $2000-2300$ & 61.5 \\
\hline Angola & Dalia & $0.15-0.4$ & 1000 & 323.15 & $21-23$ & $1-10$ & 2100 & $6-10$ \\
\hline California & Dos & 0.28 & $50-1000$ & 311.15 & 25 & $12-26$ & 1187 & $46-87$ \\
\hline Norwegian Sea & Heidrun & $0.28-0.33$ & $3000-5000$ & $358.15-361.15$ & 29 & $1.2-2.6$ & 3000 & 30 \\
\hline North Sea & Captain & $0.28-0.34$ & $5000-7000$ & 304.15 & $19-21$ & $49-200$ & 1614 & $1-84$ \\
\hline Egypt & Belayim & 0.19 & 400 & 349.15 & 20 & 8 & 3209 & 50 \\
\hline
\end{tabular}

Tabla 4. Valores de Fi según la condición dada (Evaluación de Yacimientos Prospectos para la Implementación de Procesos de Combustión In Situ Mediante Analogías, 2010).

\begin{tabular}{|c|c|c|c|}
\hline $\begin{array}{c}\text { Valor de la propiedad en el campo } \\
\text { de la base de datos }\end{array}$ & $\begin{array}{l}\text { Valor de la propiedad en el } \\
\text { campo de estudio }\end{array}$ & Condición & $\mathbf{F}_{\mathrm{i}}$ \\
\hline \multirow{5}{*}{$\begin{array}{c}\text { Rango de valores comprendidos } \\
\text { entre } \mathrm{X}_{1} \mathrm{y} \mathrm{X}_{2}\end{array}$} & \multirow{5}{*}{$\begin{array}{c}\text { Rango de valores entre } \\
\text { comprendidos entre } \mathrm{Y}_{1} \text { y } \mathrm{Y}_{2}\end{array}$} & \multirow{2}{*}{$Y_{1} \leq X_{1}<X_{2} \leq Y_{2}$} & $1-\frac{\left|X_{1}-Y_{1}\right|}{\max \left\{\left|p_{j}-p_{j e}\right|\right\}}$ si $\left|X_{1}-Y_{1}\right|>\left|Y_{2}-X_{2}\right|$ \\
\hline & & & $1-\frac{\left|X_{2}-Y_{2}\right|}{\max \left\{\left|p_{j}-p_{j e}\right|\right\}}$ si $\left|X_{1}-Y_{1}\right|<\left|Y_{2}-X_{2}\right|$ \\
\hline & & $X_{1} \leq Y_{1}<Y_{2} \leq X_{2}$ & 1 \\
\hline & & $Y_{1} \leq X_{1}<Y_{2} \leq X_{2}$ & $1-\frac{\left|X_{2}-Y_{2}\right|}{\max \left\{\mid p_{j}-p_{j e}\right\}}$ \\
\hline & & $X_{1} \leq Y_{1}<X_{2} \leq Y_{2}$ & $1-\frac{\left|X_{1}-Y_{1}\right|}{\max \left\{\mid p_{j}-p_{j e}\right\}}$ \\
\hline \multirow{3}{*}{$\begin{array}{c}\text { Rango de valores comprendidos } \\
\text { entre } \mathrm{X}_{1} \mathrm{y} \mathrm{X}_{2}\end{array}$} & \multirow{3}{*}{ Valor puntual Y } & $X_{1} \leq Y \leq X_{2}$ & 1 \\
\hline & & $X_{1}<X_{2} \leq Y$ & $1-\frac{\left|X_{2}-Y\right|}{\max \left\{\left|p_{j}-p_{j e}\right|\right\}}$ \\
\hline & & $Y \leq X_{1}<X_{2}$ & $1-\frac{\left|X_{1}-Y\right|}{\max \left\{\mid p_{j}-p_{j e}\right\}}$ \\
\hline \multirow{3}{*}{ Valor puntual X } & \multirow{3}{*}{$\begin{array}{l}\text { Rango de valores entre } \\
\text { comprendidos entre } \mathrm{Y}_{1} \text { y } \mathrm{Y}_{2}\end{array}$} & $Y_{1} \leq X \leq Y_{2}$ & $1-\frac{|\bar{X}-Y|}{\max \left\{\left|p_{j}-p_{j e}\right|\right\}}$ \\
\hline & & $X<Y_{1}<Y_{2}$ & $1-\frac{\left|X-Y_{1}\right|}{\max \left\{\mid p_{j}-p_{j e}\right\}}$ \\
\hline & & $Y_{1}<Y_{2}<X$ & $1-\frac{\left|X-Y_{2}\right|}{\max \left\{\left|p_{j}-p_{j e}\right|\right\}}$ \\
\hline
\end{tabular}

